\title{
Usefulness of Echo-Planar T2* Susceptibility-Weighted Imaging for Reliable Diagnosis of Cerebral Venous Sinus Thrombosis
}

\author{
Hidehiro Takekawa ${ }^{1}$, Hideaki Tanaka ${ }^{2}$, Tomohiro Ogawa ${ }^{1}$, Yuko Niijima ${ }^{1}$, \\ Tsubasa Sada ${ }^{2}$, Yasuhisa Daimon ${ }^{1}$ and Koichi Hirata ${ }^{2}$ \\ Key words: cerebral venous thrombosis, magnetic resonance imaging, diffusion-weighted image, diagnosis, \\ echo-planar $\mathrm{T} 2 *$ susceptibility-weighted imaging
}

(Inter Med 47: 2101-2102, 2008)

(DOI: 10.2169/internalmedicine.47.1588)

A 38-year-old man experienced "thunderclap" headache. Six days later he presented to our hospital with dysarthria. Brain computed tomography (CT), T1- and T2-weighted images revealed no abnormalities, but abnormal intensity areas were detected on DWI (diffusion-weighted image) and T2* SW (echo-planar T2* susceptibility-weighted imaging) (Picture 1). Subsequently, we performed MR (magnetic resonance) venography and he was confirmed to have CVT (cerebral venous sinus thrombosis) of the superior sagittal sinus with protein $\mathrm{C}$ deficiency.

CT diagnosis of CVT is difficult and there is a high false negative rate of $52.6 \%$ (1). Idbaih et al (2) reported diagnostic superiority of MR imaging, in which $\mathrm{T} 2 * \mathrm{SW}$ could easily detect abnormalities at the early stage rather than DWI.

CVT is a rare condition which is notoriously difficult to diagnose due to its variable modes of onset and its wide spectrum of signs and symptoms (3). We propose $\mathrm{T} 2 * \mathrm{SW}$ for expeditious diagnosis of considerable CVT.

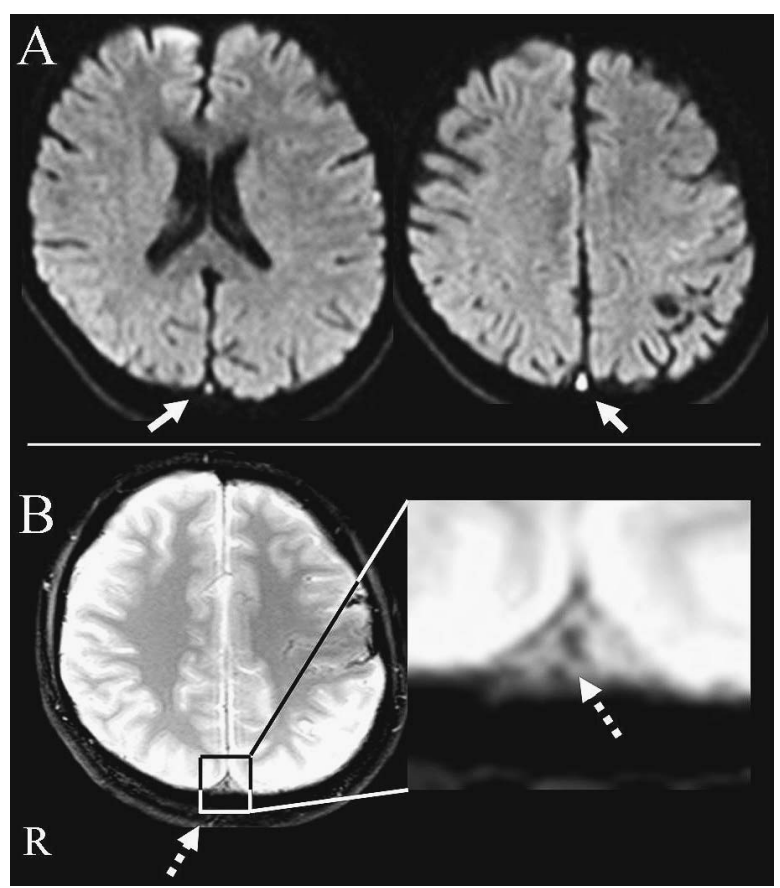

Picture 1. Magnetic resonance images of cerebral venous thrombosis A: The high-signal area suggestive of the existence of venous thrombosis of the superior sagittal sinus was detected on diffusion-weighted image (ST: 6.00, TR: 6000.00, TE: 85.00) (arrows). B: The low-intensity signal was detected on echo-planar $\mathrm{T}^{*} *$ susceptibility-weighted imaging in the same area of the superior sagittal sinus indicated by diffusionweighted image (ST: 6.00, TR: 680.00, TE: 20.00) (dotted arrows). However, conventional T1/T2-weighted image and fluid-attenuated inversion recovery revealed no abnormalities in the same area of the superior sagittal sinus.

${ }^{1}$ Stroke Division, Department of Neurology, Dokkyo Medical University, Tochigi and ${ }^{2}$ Headache Division, Department of Neurology, Dokkyo Medical University, Tochigi

Received for publication August 13, 2008; Accepted for publication September 1, 2008

Correspondence to Dr. Hidehiro Takekawa, takehide@k8.dion.ne.jp 
Inter Med 47: 2101-2102, 2008 DOI: 10.2169/internalmedicine.47.1588

\section{References}

1. Tang PH, Chai J, Chan YH, Chng SM, Lim CC. Superior sagittal sinus thrombosis: subtle signs on neuroimaging. Ann Acad Med Singapore 37: 397-401, 2008.

2. Idbaih A, Boukobza M, Crassard I, Porcher R, Bousser MG, Chabriat H. MRI of clot in cerebral venous thrombosis: high diag- nostic value of susceptibility-weighted images. Stroke 37: 991995, 2006.

3. Ameri A, Bousser MG. Cerebral venous thrombosis. Neurol Clin 10: 87-111, 1992.

\section{(C) 2008 The Japanese Society of Internal Medicine http://www.naika.or.jp/imindex.html}

